\title{
Separation of the Systems for Color and Spatial Manipulation in Working Memory Revealed by a Dual-task Procedure
}

\author{
Harald M. Mohr ${ }^{1}$ and David E. J. Linden ${ }^{1,2,3}$
}

\begin{abstract}
- The manipulation of different kinds of content is fundamental to working memory. It has been suggested that the mere maintenance of color and spatial information occurs in parallel, but little is known about whether this holds true for manipulation as well. Using a dual-task delayed-response paradigm that required the manipulation of color and angles, this study finds that the two functions do not interfere. Conversely, interference did occur when both components of a dual-task tapped into the spatial
\end{abstract}

\section{INTRODUCTION}

Different streams that are spatially segregated subserve the transmission of visual information from the retina to the primary visual cortex (Livingstone \& Hubel, 1987). This separation of processing streams continues beyond the primary visual cortex. Ungerleider and Mishkin (1982) proposed a ventral "what" and a dorsal "where" pathway of higher visual processing. The ventral pathway is mostly associated with feature analysis (e.g., texture, color) and object recognition and extends from $\mathrm{V} 1$ and V2 via V4 into the inferior temporal cortex. The dorsal pathway is involved in motion analysis, spatial relations, and visually guided action (Goodale, 1998) and extends from V1 and V2 to the human motion complex MT (Goebel, Khorram-Sefat, Muckli, Hacker, \& Singer, 1998) and parietal areas (Sakata, Taira, Kusunoki, Murata, \& Tanaka, 1997).

The role of these distinct functional pathways in the processing of visual information and its attentional control has been investigated extensively (Ungerleider \& Haxby, 1994). It has furthermore been suggested that the ventral/dorsal stream distinction also has an effect on the maintenance of visual object and spatial information in working memory (WM) (Munk et al., 2002; Ungerleider, Courtney, \& Haxby, 1998; Goldman-Rakic, 1996). WM is a system for short-term maintenance and manipulation of information (Baddeley, 1992). According to

${ }^{1}$ Johann Wolfgang Goethe-Universität, Germany, ${ }^{2}$ Max Planck Institute for Brain Research, Gemany, ${ }^{3}$ University of Wales system. Thus, color and spatial information are manipulated in parallel. A concurrent phonological task did not interfere with either maintenance or manipulation, whereas a task requiring central executive processes interfered with manipulation only. We speculate that the ventral-dorsal dissociation of visual processing is conserved for manipulation processes and that manipulation differs from maintenance in the extent to which is relies on central executive resources.

Baddeley and Hitch (1974), the WM system consists of a central executive (CE) and two subsidiary systems, the phonological loop and the visual-spatial sketchpad, rehearsing phonological and visual-spatial information. The CE is considered to be a general attention control system to which a wide range of high-level cognitive functions are attributed (Baddeley, 1996).

Visual WM cannot be regarded as a unique, homogenous system, but rather as comprising different and at least partially independent subcomponents (Pickering, 2001; Logie, 1995). Neuropsychological cases and experimental studies support a separation within visual WM that largely corresponds to the differentiation of the perceptual processing streams. In this sense, visual information would be bifurcated into visual ("what") and spatial ("where") information. Patient MV, who had suffered a right frontal ischemic lesion, showed impaired performance of spatial WM tasks, but not of tasks that require form analysis or verbal WM (Carlesimo, Perri, Turriziani, Tomaiuolo, \& Caltagirone, 2001). Della Sala, Gray, Baddeley, Allamano, and Wilson (1999) described a double dissociation in brain-damaged patients when they completed the visual pattern test (visual components) and the Corsi-block test (spatial components).

A dissociation of visual and spatial components in visual WM is also suggested by experimental studies that used the dual-task design. The dual-task methodology hinges on the idea that if two tasks can be performed simultaneously without producing a substantial drop in performance, these tasks can be assumed independent. 
Dual-task studies showed impaired performance of spatial processing tasks when a movement discrimination task had to be completed at the same time, but not of a color discrimination task, whereas object WM tasks showed the opposite pattern of interference (Tresch, Sinnamon, \& Seamon, 1993; Logie \& Marchetti, 1991). The separation of the maintenance of visual and spatial information in WM was replicated in a number of studies with similar interference experiments (Della Sala et al., 1999; Kessels, Postma, \& de Haan, 1999; Hecker \& Mapperson, 1997).

However, the separation between visual and spatial components does not constitute the only possible differentiation within visual WM. Theoretical considerations and empirical findings also suggest a distinction between passive and active processes. Passive processes are recruited by tasks that require participants to recall information in the same format as it was memorized (maintenance or storage of information). Active processes are recruited by tasks that require participants to modify, transform, integrate, or otherwise manipulate information. Support for the view of separate passive and active processes in visual WM came from neuropsychological case studies (Cornoldi, Rigoni, Venneri, \& Vecchi, 2000; Morton \& Morris, 1995). Cornoldi et al. (2000) reported a double dissociation of passive and active visual WM performance between two children suffering from nonverbal learning disability. One child performed well on storage tasks such as the Corsi-block test and visual pattern test, but performed poorly on mental rotation tasks, which required active manipulation of the stored information. The other child showed the converse pattern of results. Additional support for the separation of active and passive processes comes from sex comparison studies (Vecchi \& Girelli, 1998), comparisons between blind and sighted (Vecchi, 1998), and between young and elderly individuals (Vecchi \& Cornoldi, 1999), and from functional neuroimaging (D'Esposito, Postle, \& Rypma, 2000). The results of the dual-task paradigms also suggest that active and passive processes in visual WM are based on different neural mechanisms. Bruyer and Scailquin (1998) found evidence that only manipulation, but not maintenance, of spatial information shares resources with CE processes. The reported studies thus indicate that visual WM is not a unitary system. It can be subdivided according to the type of information into a visual (form, color, and texture) and spatial domain (spatial relationship, movements) and according to the processes needed for the task (maintenance vs. manipulation).

The series of experiments described in this article served two main purposes. We aimed to reveal the extent to which the manipulation processes of visual WM are segregated according to the type of information, and determine behavioral characteristics that distinguish them from maintenance processes. To test the independence of the visual and the spatial domain, we designed a dual-task paradigm with color (probing the ventral stream) and spatial (probing the dorsal stream) manipulation. We assumed that interference between two tasks would manifest itself in significantly lower signal detection sensitivity in the dual condition compared to the more difficult of the single conditions. We hypothesized that interference would not occur between color and spatial manipulation because they are supported by separate neural subsystems with distinct resources but only in a control experiment with two manipulation tasks that both tapped into the spatial subsystem of WM.

To demonstrate that manipulation and maintenance are based on different mechanisms, we engaged the phonological loop, by an articulatory suppression task (Levy, 1977), and CE processes, by a random word generation task (Baddeley, 1966), during manipulation and maintenance. The random word generation task was expected to interfere only with manipulation but not with maintenance because only the former relies heavily on CE resources (Bruyer \& Scailquin, 1998). Articulatory suppression should have no interference effect on either manipulation or maintenance because the main tasks are not supposed to involve verbally codable material.

\section{RESULTS}

\section{Color-Spatial Interference}

Signal detection models were used to analyze the data. $A^{\prime}$ scores were calculated as measure of signal detection sensitivity (Grier, 1971) to detect an interference effect. $A^{\prime}$ increases from 0.5 for chance performance to 1 for perfect performance. $A^{\prime}$ was used instead of $d^{\prime}$ because $A^{\prime}$ is more robust against violations of the assumption that the variances of the hypothetical distributions are equal (Donaldson, 1993) and $A^{\prime}$ does not suffer from the indeterminacy of $d^{\prime}$ that occurs in the absence of false alarms. $A^{\prime}$ estimates the area under the receiver operating curve. $A^{\prime}$ was calculated for each participant following the formula by Grier (1971):

$A^{\prime}=0.5+[(H-F A) \times(1+H-F A)] /[4 \times H \times(1-F A)]$

where $H$ (hit) is the correct detection of matching trial and $F A$ (false alarm) is the nonmatching trial identified as matching trial.

If $F A>H$, the point lies beyond the chance diagonal and the following formula is used:

$A^{\prime}=0.5-[(F A-H) \times(1+F A-H)] /[4 \times F A \times(1-H)]$

One-way analyses of variance (ANOVAs, repeated measurement) were calculated to compare the three experimental conditions ( $\alpha=5 \%$, both-sided). A single contrast (Helmert) was calculated for the single condition with the lowest $A^{\prime}$ and the dual-task condition $(\alpha=$ 
Figure 1. (1) Example of a trial in Experiment 1. Two samples were presented for $500 \mathrm{msec}$ and then, after a 500-msec delay, the instruction was shown for $1500 \mathrm{msec}$. This was followed by a white fixation cross (2000 msec) and the test stimulus, which was presented for 3000 msec. Feedback was given by a change of the color of the fixation cross. In the intertrial interval (ITI, $2000 \mathrm{msec}$ ), a white fixation cross was shown. Participants had to mix color, rotate the semicircle, or perform both tasks as a dual task (Experiment 1, 2, and 3). (2) In Experiment 2 , the test stimulus was presented simultaneously with the samples $(3000 \mathrm{msec})$.

Feedback was given afterward (1000 msec). The instruction was shown for $1500 \mathrm{msec}$ before the stimuli. In the ITI, a white fixation cross was presented (2000 $\mathrm{msec})$. (3) In Experiment 3, the instruction was presented at the beginning of each trial (1500 msec), followed by the first sample (600 msec). After a delay of $400 \mathrm{msec}$, the second sample was shown simultaneously with the test stimulus $(3500 \mathrm{msec})$ Feedback was given immediately afterward (1000 msec). In the ITI, a white fixation cross was presented $(2000 \mathrm{msec})$. (4) In Experiment 4 , the test stimulus was presented simultaneously with the samples $(3000 \mathrm{msec})$. Feedback was given afterward $(1000 \mathrm{msec})$. The instruction was shown for $1500 \mathrm{msec}$ before the stimuli. In the ITI, a white fixation cross was presented $(2000 \mathrm{msec})$. Participants had to rotate the semicircle, to compare distances, or perform both tasks concurrently.

$5 \%$, both-sided). This approach was chosen because the dual task involved both transformations that were required in the single tasks, and thus, under the assumption of shared resources (which we only made in
Experiment 4), should be more demanding than the more difficult of the single tasks. Differences in reaction time (RT) between the experimental conditions for all experiments were also calculated (one-way ANOVA,
Figure 4. (1) Example of a trial in Experiment 5. Two samples were presented for $500 \mathrm{msec}$ and then, after $1500 \mathrm{msec}$ delay, the test stimulus was presented $(3000 \mathrm{msec})$. Feedback was given afterward (1000 msec). In the ITI, a white fixation cross was presented $(2000 \mathrm{msec})$. Task: Participants had to mix color or rotation angle, without interference (NI), while performing an articulatory suppression task (AS) or while performing a random generation task (RG). (2) In Experiment 6, two samples were presented $(500 \mathrm{msec})$, and then, after a 1500-msec delay, two test stimuli were shown (3000 msec). Feedback was given afterward (1000 msec). In the ITI, a white fixation cross was presented $(2000 \mathrm{msec})$. Task: Participants had to maintain the color or rotation of both semicircles. Interference conditions were as above.

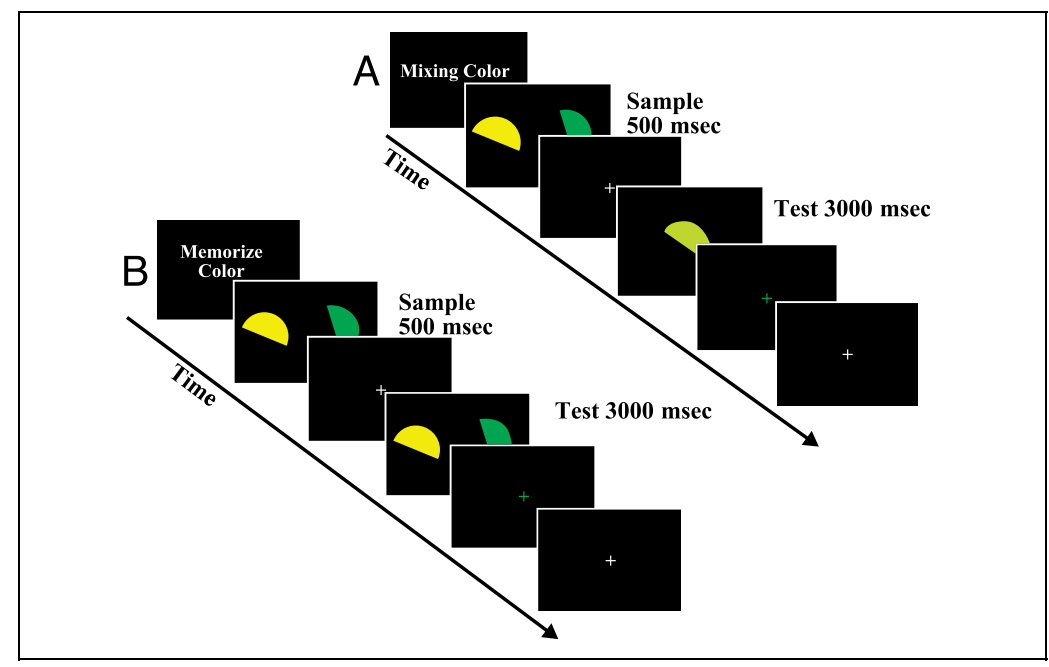


Figure 2. Mean $A^{\prime}$ and $S E$ of Experiments 1-4 for the three experimental conditions [Experiments 1-3: spatial manipulation (spatial), color manipulation (color), and dual manipulation (dual), Experiment 4: spatial manipulation 1 (spatial 1) spatial manipulation 2 (spatial 2), and dual manipulation (dual)]. The dual condition marked with an asterisk is significantly $(p<.05)$ lower in $A^{\prime}$ in comparison to the single condition with lowest $A^{\prime}$ score.

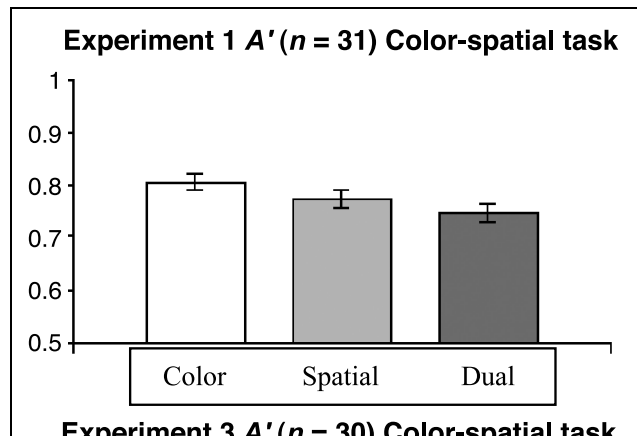

Experiment $3 A^{\prime}(n=30)$ Color-spatial task

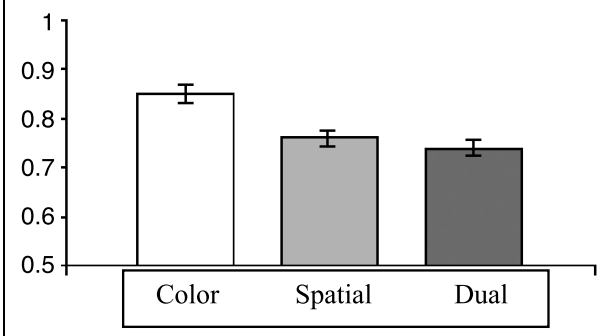

Experiment $2 A^{\prime}(n=11)$ Color-spatial task

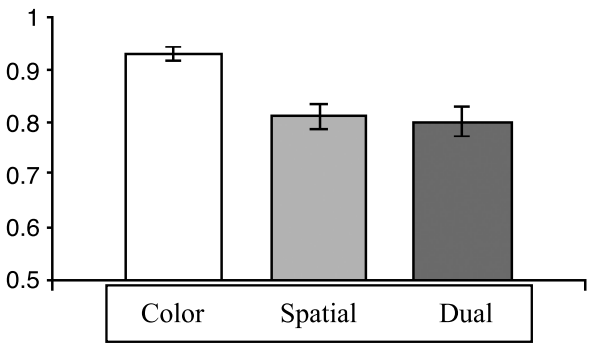

Experiment $4 A^{\prime}(n=22)$ Spatial-spatial task

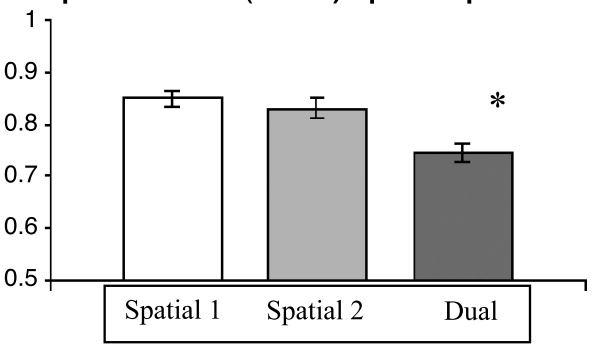

repeated measurement) to exclude that the dual-task condition was solved serially.

In Experiment 1, the color manipulation task required participants to judge if the color of a semicircle (test) was the result of mixing the colors of two previously presented semicircles (samples). The spatial task was based on the same visual material but participants had to judge whether the test stimulus was in the middle rotation angle of the samples (Figure 1). In the dual-task condition, participants had to assess both the color and

Table 1. Mean $(S E)$ of Hit Rate $(H)$, False Alarm Rate $(F A)$, and $A^{\prime}$ Scores for the Three Experimental Conditions for Experiments $1-4$

Single Contrast Dual vs.

$H \quad F A \quad A^{\prime} \quad$ Main Effect $A^{\prime} \quad$ Single Condition with Lowest $A^{\prime}$

Experiment 1

$\begin{array}{lccr}\text { Color } & 0.74(0.03) & 0.3(0.02) & 0.80(0.01) \\ \text { Spatial } & 0.7(0.3) & 0.31(0.01) & .77(0.02) \\ \text { Dual } & 0.68(0.03) & 0.34(0.02) & 0.74(0.02)\end{array}$

Experiment 2

$\begin{array}{lccccc}\text { Color } & 0.81(0.05) & 0.06(0.05) & 0.93(0.01) & F(2,20)=16.52, p<.001 & n s \\ \text { Spatial } & 0.68(0.04) & 0.23(0.03) & 0.81(0.02) & & \\ \text { Dual } & 0.7(0.05) & 0.23(0.01) & 0.8(0.03) & \\ \text { Experiment 3 } & & & & & \\ \text { Color } & 0.75(0.03) & 0.19(0.02) & 0.85(0.02) & F(2,58)=15.5, p<.001 & \\ \text { Spatial } & 0.69(0.02) & 0.3(0.03) & 0.76(0.02) & & \\ \text { Dual } & 0.67(0.03) & 0.34(0.02) & 0.74(0.02) & & \\ \text { Experiment } 4 & & & & \\ \text { Spatial 1 } & 0.72(0.03) & 0.17(0.02) & 0.85(0.01) & F(2,42)=5.84, p=.006 \\ \text { Spatial 2 } & 0.72(0.03) & 0.19(0.02) & 0.83(0.02) & \\ \text { Dual } & 0.63(0.03) & 0.25(0.02) & 0.76(0.01) & \end{array}$

Comparisons of $A^{\prime}$ scores between the three experimental conditions of experiments 1, 2, 3, and 4 with one-way ANOVA (repeated measurements) and with single contrast (comparison dual condition vs. single task with lowest $A^{\prime}$ score). 
Figure 3. Mean RT (hits) and $S E$ of Experiments 1-4 for the three experimental conditions.

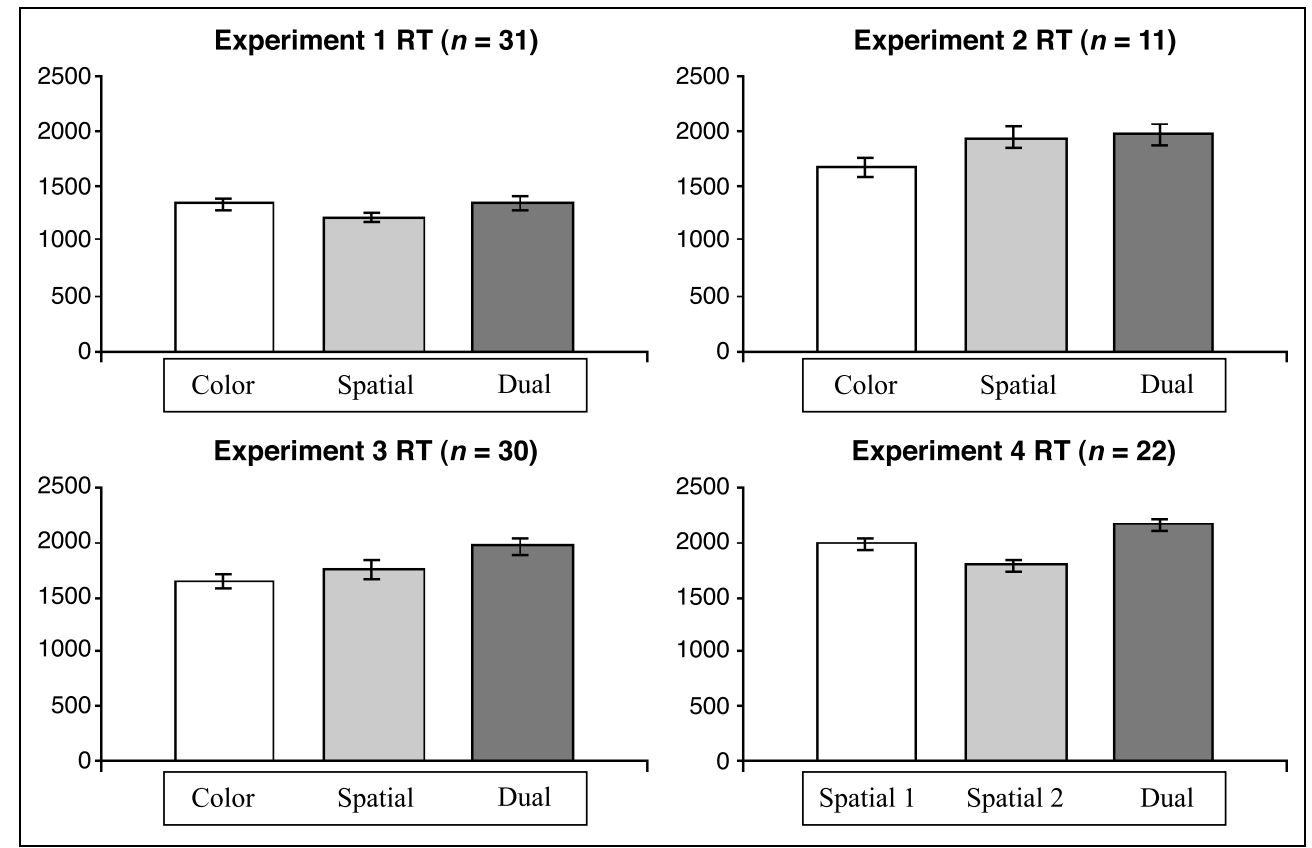

the angle. The mean $H, F A$, and $A^{\prime}$ scores $( \pm S E)$ for the three conditions are depicted in Figure 2 and Table 1. The main effect for $A^{\prime}$ was significant for the three conditions, $F(2,60)=3.28, p=.044$. No significant difference was found for the single condition with the lowest $A^{\prime}$ (spatial manipulation) and the dual condition, $F(1,30)=0.93 p=.34$. The one-way ANOVA (repeated measurement) showed a significant overall effect, $F(2,60)=15.9, p<.01$, for the three experimental conditions in the RT, but no significant difference was found for the single condition with the highest RT and the dual-task condition, $F(1,30)=0.018, p=.89$. RTs are presented in Figure 3.

Our hypotheses were thus confirmed in Experiment 1. There was no interference in the dual condition with respect to the single task conditions. This suggests that, as hypothesized, two independent resources are available for color and spatial manipulation. In the dual conditions of Experiments 1, the RTs were close to those of the more difficult of the single conditions. The RT results thus support the notion that participants solved the dual task in an essentially parallel manner.

\section{Relying on Serial Processing or Gestalt Cues}

One possible criticism of the results from Experiment 1 is that the delay period between samples and tests in Experiment 1 might have allowed enough time to perform the dual condition in serial order, thus, obscuring potential RT effects. Moreover, in Experiment 1, the two sample semicircles were presented simultaneously. One may contend that participants might have solved the spatial task by mentally extending the base of each semicircle until the lines met and then dividing the resulting angle.
Experiments 2 and 3 were designed to exclude these possibilities. In Experiment 2, there was no gap between sample and test to preclude a serial strategy for solving the task. In Experiment 3, only one stimulus was shown at the initial sample presentation, whereas the second sample was shown together with the test stimulus (Figure 1). The mean $H, F A, A^{\prime}$, and RT for the three conditions are shown in Figures 2 and 3 and Table 1. The analysis of the $A^{\prime}$ score showed a significant main effect for the three conditions in Experiments 2 and 3 [Experiment 2, $F(2,20)=16.52, p<.001$; Experiment 3, $F(2,58)=15.5, p<.001]$ but no significant difference between the single condition with the lowest $A^{\prime}$ score and the dual condition for either experiment [Experiment $2, F(1,10)=0.03, p=.85$; Experiment $3, F(1,29)=$ 1.97, $p=.17]$.

The one-way ANOVA (repeated measurement) showed a significant overall effect in Experiments 2 and 3 for RT [Experiment 2, $F(2,20)=12.34, p<.001$; Experiment 3, $F(2,58)=15.74, p<.01]$. There was no significant difference between the single condition with the highest RT and the dual condition in Experiment $2[F(1,10)=$ $0.05, p=.81]$ and only a trend in Experiment $3[F(1,29)=$ $3.8, p=.06]$.

Experiments 2 and 3 thus also support our hypothesis that the manipulation of "what" information and the manipulation of "where" information are performed by separate active systems in WM. The time structure of stimulus presentation in Experiments 2 and 3 prevented participants from solving the dual task in serial order (no gap between last sample and test). In the dual conditions of both experiments, the RT were close to those of the more difficult of the single conditions, and there was no indication that reaction times would add up in any case. Thus, the RT results also support the 
interpretation that participants successfully performed the component tasks of the dual condition in parallel. Furthermore, there was no evidence that the lack of interference in Experiment 1 resulted from participants solving the spatial manipulation task by gestalt perception cues.

\section{Spatial-Spatial Interference}

Experiment 4 was a spatial-spatial dual task (Figure 1). This experiment was included to show that WM manipulation tasks relying on the same domain of visual processing do indeed interfere with each other. Here, a decline of signal detection sensitivity $\left(A^{\prime}\right)$ was expected for the dual condition because both spatial manipulation tasks are supported by the same spatial subsystem of visual WM. Figure 2 and Table 1 show the results for $H$, $F A$, and the $A^{\prime}$ score of Experiment 4. A significant main effect was found for the three conditions, $F(2,42)=$ 5.84, $p<.01$, and a significantly lower $A^{\prime}$ score was demonstrated in the dual-task condition compared to the single condition with the lowest $A^{\prime}$ score (spatial 2, distance judgment), $F(1,21)=5.83, p<.05$.

In Experiment 4 we observed interference in the dualtask condition as measured by $A^{\prime}$ score and hit rate in comparison to the single task with lowest $A^{\prime}$ or hit rate. The one-way ANOVA (repeated measurement) also showed a significant overall effect, $F(2,42)=47.75$, $p<.01$, on RT and a significant difference between the single condition with the highest RT and the dual condition, $F(1,21)=18.59, p<.01$ (Figure 3 ).

Our hypothesis was thus confirmed in Experiment 4. We demonstrated that a dual task performed on the same visual material as the single task but involving multiple processes in the same cognitive domain is in fact characterized by an interference effect. Because the absence of interference in Experiments 1, 2, and 3 might have been the result of a floor effect, it was important to rule out this alternative interpretation by including a task with the same difficulty where we did observe interference in the dual task.

\section{Differences between Manipulation and Maintenance}

We assume that manipulation requires CE resources. Thus, random generation is expected to interfere with both manipulation tasks when compared to the control condition. Articulatory suppression should have no interference effect on the manipulation tasks because task solving is not aided by verbalization, with very similar colors being used for both sample and test stimuli. In Experiment 5, participants performed the color and the spatial manipulation task (Figure 4) in three dual-task conditions. Condition NI (no interference) did not include any additional task (control), whereas condition AS included articulatory suppression and condition RG included random generation of strings of letters. Experiment 6 was similar to Experiment 5, with the exception that participants performed a color and orientation maintenance task.

Results of Experiment 5 are presented in Figure 5 and in Table 2. Two one-way ANOVAs (repeated measurement) with three levels (NI, AS, and RG) were calculated for spatial and color manipulation. The analysis of the $A^{\prime}$ scores showed a significant main effect of the three conditions (NI, AS, and RG) for spatial manipulation $(F(2,58)=7.53, p<.01)$ and for color manipulation $(F(2,58)=3.68, p<.05)$. Single contrasts were calculated to compare spatial or color manipulation in the NI condition with that in the AS or RG conditions. For spatial manipulation, no significant difference $A^{\prime}$ was found between NI and AS, $F(1,29)=0.036, p=.85$. However, the difference between NI and RG was significant, $F(1,29)=9.38, p<.01$. For color manipulation, the single contrasts likewise showed a significant difference between NI and RG, $F(1,29)=15.57, p<.01$, but not between NI and AS, $F(1,29)=0.71, p=.4$. Both manipulation tasks, thus, were subject to interference from the random generation task, but not from articulatory suppression. The RT for the manipulation of color showed a significant overall effect, $F(2,58)=4.5, p=$ .016 (one-way ANOVA, three levels, repeated measurement) and a significant difference between NI and RG in the single contrast, $F(1,29)=6, p<.05$. The RT of the spatial manipulation task showed a significant overall effect, $F(2,58)=4.1, p<.05$, and a significant difference between NI and AS in the single contrast, $F(1,29)=5.33$, $p<.05$ (Figure 6).

As hypothesized, the random generation task interfered with the manipulation of color and spatial information. The results support the assumption that processes of manipulation rely on $\mathrm{CE}$ resources to a sufficient extent. In agreement with the hypothesis that our tasks did not involve verbally codable material, the articulatory suppression task did not interfere with the manipulation of either type of information.

In contrast to manipulation, maintenance is not assumed to rely on CE resources. Therefore, when compared to the control condition, random generation is not expected to interfere with the maintenance tasks. The results of Experiment 6 are shown in Figure 5 and in Table 2. Two one-way ANOVAs (repeated measurement) with three levels (NI, AS, and RG) were calculated for spatial maintenance and color maintenance. The analysis of the $A^{\prime}$ scores showed no significant main effect of the three conditions (NI, AS, and RG) for spatial maintenance, $F(2,58)=0.78, p=.46$, and for color maintenance, $F(2,58)=0.09, p=.9$ (Table 2). Thus, neither maintenance task was subject to interference from random generation or articulatory suppression. The RT for the maintenance of color showed no significant difference, $F(2,58)=0.55, p=.57$ (one-way ANOVA, three levels, repeated measurement). The RT of the spatial 
Figure 5. Experiment 4: Mean $A^{\prime}$ scores and $S E$ for (a) color and spatial manipulation (Experiment 5) and (b) color and spatial maintenance (Experiment 6) in the three experimental conditions, NI (control), AS, and RG. Conditions marked with asterisks are significantly $(p<.05)$ different from the control condition.
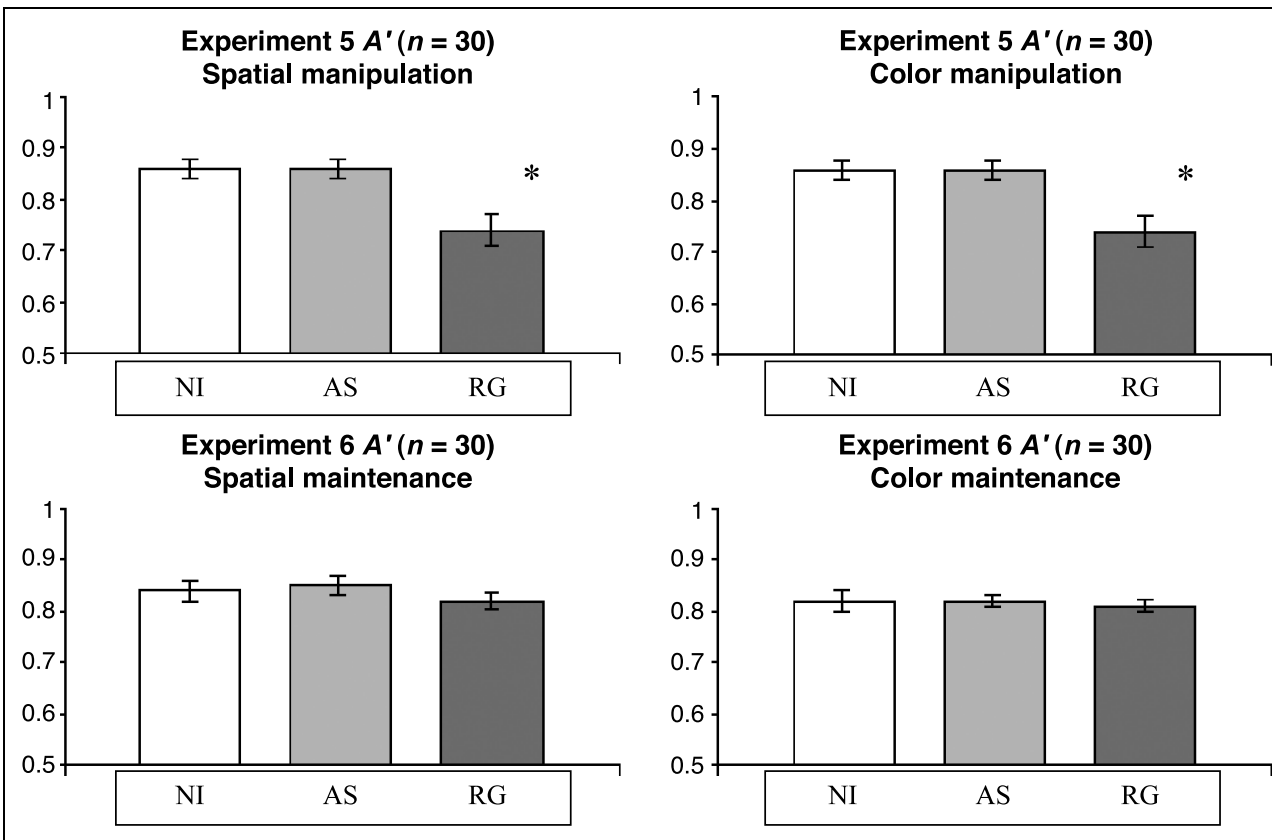

Experiment $6 A^{\prime}(n=30)$ Color maintenance

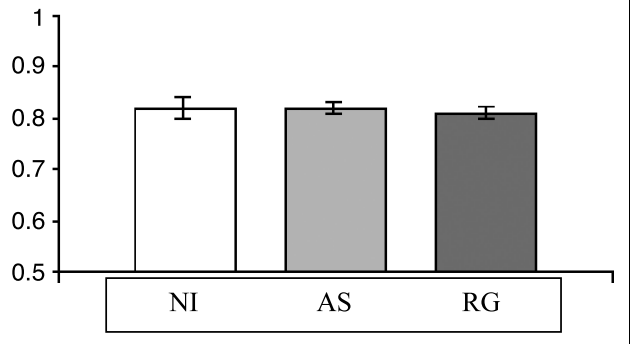

maintenance task showed an overall significant difference, $F(2,58)=4.4, p=.01$, and a trend for the difference between NI and RG in the single contrast, $F(1,29)=3.26, p=.08$ (Figure 6).
As hypothesized, the random generation task did not interfere with the maintenance of color or spatial information. The results support the assumption that a CE contribution is not crucial for processes of maintenance.

Table 2. Mean $(S E)$ of Hit Rate $(H)$, False Alarm Rate $(F A)$, and $A^{\prime}$ Score for the Three Experimental Conditions for Experiments 5 and 6

\begin{tabular}{|c|c|c|c|c|c|}
\hline & $H$ & $F A$ & $A^{\prime}$ & Main Effect $A^{\prime}$ & $\begin{array}{c}\text { Single Contrasts } A^{\prime} \text { : (1) NI vs. } \\
A S \text { and (2) NI vs. RG }\end{array}$ \\
\hline \multicolumn{6}{|c|}{ Experiment 5} \\
\hline Spatial NI & $0.86(0.03)$ & $0.28(0.03)$ & $0.86(0.02)$ & $F(2,58)=7.53, p=.001$ & (1) $n s$ \\
\hline Spatial AS & $0.86(0.02)$ & $0.37(0.03)$ & $0.86(0.04)$ & & (2) $F(1,29)=9.38, p=.005$ \\
\hline Spatial RG & $0.73(0.05)$ & $0.38(0.03)$ & $0.74(0.02)$ & & \\
\hline \multicolumn{6}{|c|}{ Experiment 5} \\
\hline Color NI & $0.77(0.03)$ & $0.33(0.03)$ & $0.80(0.03)$ & $F(2,58)=3.68, p=.031$ & (1) $n s$ \\
\hline Color AS & $0.73(0.03)$ & $0.34(0.02)$ & $0.76(0.03)$ & & (2) $F(1,29)=15.57, p<.01$ \\
\hline Color RG & $0.63(0.05)$ & $0.38(0.03)$ & $0.69(0.03)$ & & \\
\hline \multicolumn{6}{|c|}{ Experiment 6} \\
\hline Spatial NI & $0.8(0.03)$ & $0.26(0.02)$ & $0.84(0.02)$ & ns & (1) $n s$ \\
\hline Spatial AS & $0.81(0.03)$ & $0.26(0.02)$ & $0.85(0.02)$ & & (2) $n s$ \\
\hline Spatial RG & $0.73(0.04)$ & $0.28(0.03)$ & $0.82(0.01)$ & & \\
\hline \multicolumn{6}{|c|}{ Experiment 6} \\
\hline Color NI & $0.85(0.02)$ & $0.39(0.03)$ & $0.82(0.02)$ & ns & (1) $n s$ \\
\hline Color AS & $0.83(0.02)$ & $0.37(0.03)$ & $0.81(0.02)$ & & (2) $n s$ \\
\hline Color RG & $0.75(0.03)$ & $0.28(0.02)$ & $0.81(0.02)$ & & \\
\hline
\end{tabular}

Comparisons of $A^{\prime}$ scores for experiments 5 and 6 among the three conditions NI (Control), AS, and RG (one-way repeated measurement ANOVAs with two single contrasts: NI vs. AS and NI vs. RG). 
Figure 6. Mean RT (hits) and $S E$ for (a) color and spatial manipulation (Experiment 5) and (b) color and spatial maintenance (Experiment 6) in the three experimental conditions, NI (control), AS, and RG.

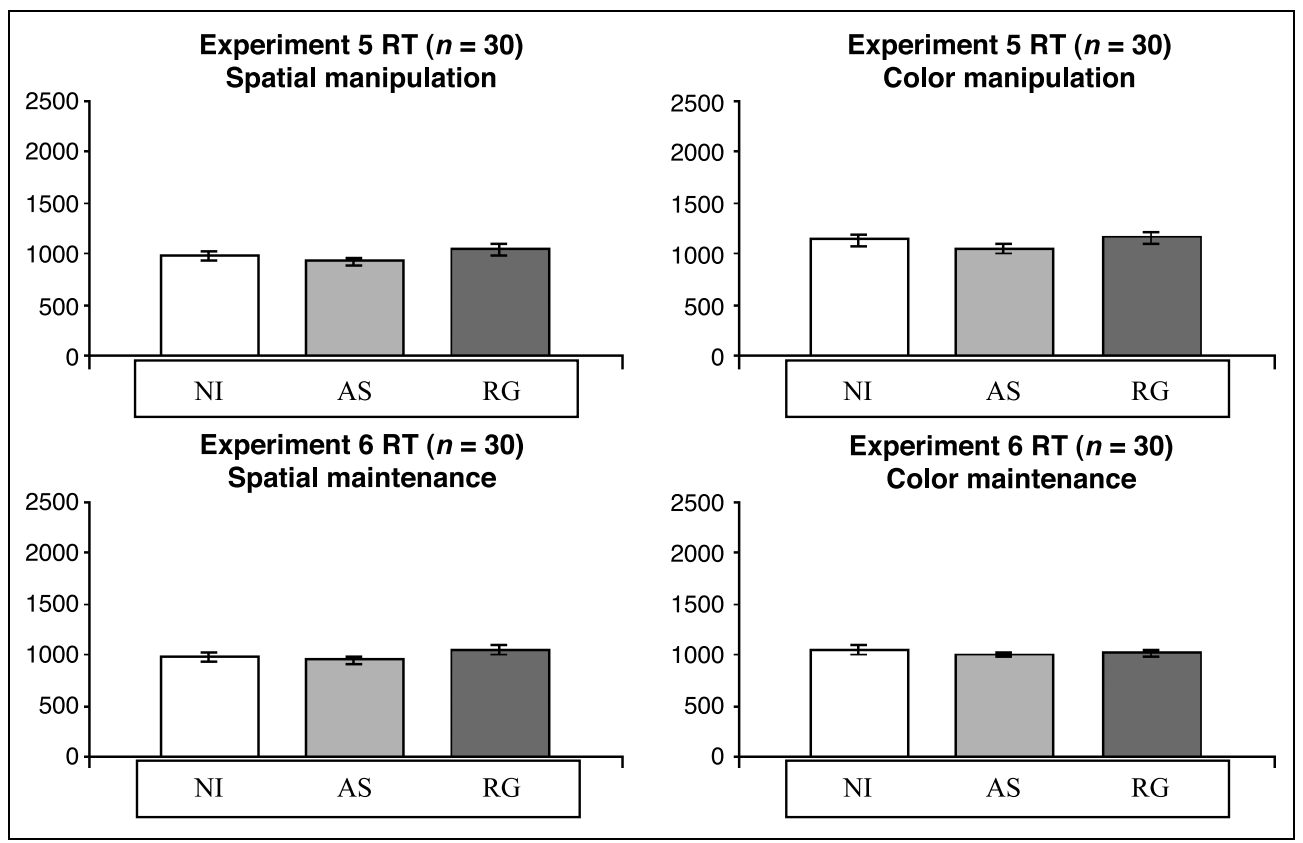

In agreement with the hypothesis that our tasks did not involve verbally codable material, the articulatory suppression task did not interfere with the manipulation of either type of information.

\section{DISCUSSION}

The study was designed to test the assumption that processes of manipulation in visual WM are separable into a spatial and a visual component and to compare the mechanisms of manipulation and maintenance. In the first step, a dual-task paradigm was used in which the participants were asked mentally to mix colors and manipulate spatial information separately as single tasks, or simultaneously as a dual task (Experiments 1, 2, and 3 ), or to solve two spatial manipulation tasks simultaneously (Experiment 4). We hypothesized that Experiments 1, 2, and 3 would show no interference between color and spatial manipulation in the dual condition because these processes rely on separate subsystems with distinct resources, whereas Experiment 4 would show interference between the two spatial tasks because they both require access to resources of the spatial subsystem of visual WM.

Two further dual-task experiments were designed to compare manipulation and maintenance processes. Participants performed a color and a spatial manipulation (Experiment 5) or maintenance task (Experiment 6) in different dual-task conditions (NI, AS, and RG). In NI, they performed the WM task without any second task (control), in AS, with articulatory suppression, and in $\mathrm{RG}$, with random vocal generation of strings of letters. It was hypothesized that RG would interfere with the manipulation, but not the maintenance task.
Our hypotheses were confirmed in all experiments. In sum, Experiments 1-4 support the assumption that color and spatial manipulation in WM rely on different resources. Experiments 5 and 6 suggest that maintenance and manipulation differ in the extent to which they require CE resources. Most experiments that demonstrated a dissociation of dorsal and ventral processing streams of visual WM used passive tasks like maintenance or storage of information (Della Sala et al., 1999; Kessels et al., 1999; Hecker \& Mapperson, 1997; Tresch et al., 1993; Logie \& Marchetti, 1991). The present study extends findings of a ventral-dorsal dissociation in visual WM to active manipulation of information. Until now, only Vuontela et al. (1999) used a paradigm that actively taps CE functions ( $n$-back task). The results of this study indicated that active processes, too, could be differentiated into visual and spatial components. However, the $n$-back task does not require a domain-specific transformation of the memorized objects (but rather a continuous updating of their position in the train of stimuli), and a study by Meegan, Purc-Stephenson, Honsberger, and Topan (2004) suggests that the $n$-back task is not sensitive to differences between spatial and nonspatial information.

\section{Distinct Systems in Visual Working Memory}

Supporting evidence for a segregation of the active part of visual WM according to the type of information comes from neuropsychological case studies that showed that impairments in visual and spatial imagery could exist independently (Luzzatti, Vecchi, Agazzi, Cesa-Bianchi, \& Vergani, 1998; Farah, Hammond, Levine, \& Calvanio, 1988). Generation of mental images shares many ele- 
ments with an active WM task (Formisano et al., 2002). Thus, studies that demonstrate a dissociation of visual and spatial mental imagery are also suggestive of a bimodal manipulation system in WM. The differentiation of visual WM into partly segregated manipulation and maintenance processes is also supported by the present results. Our results are consistent with the findings of Bruyer and Scailquin (1998), who demonstrated that spatial manipulation, but not maintenance tasks require CE resources.

\section{Functional Subdivision of Prefrontal Cortex for Visual WM}

This subdivision of visual WM according to the type of material (ventral vs. dorsal) and the process (manipulation vs. maintenance) is also supported by anatomical and electrophysiological studies in animals and functional neuroimaging studies in humans. These studies have resulted in the "domain-specific" and "process-specific" theories of frontal cortex (FC) organization. According to the domain-specific theory, the ventrolateral frontal cortex (VLFC) would be responsible for the maintenance of information from the ventral visual processing stream, whereas the dorsolateral frontal cortex (DLFC) is believed to be responsible for the maintenance of information from the dorsal visual processing stream (Goldman-Rakic, 1987). This theory is mainly based on lesion and electrophysiological studies in nonhuman primates.

The process-specific theory implies that the FC is functionally segregated according to the nature of processing performed, with the VLFC specialized for the maintenance and matching of information and the DLFC specialized for active manipulation and monitoring (Petrides, 1994). The literature presents a mixed picture with respect to these two theories. Although some functional imaging studies of human visual WM produced results consistent with the domain-specific theory (Courtney, Petit, Haxby, \& Ungerleider, 1998), most studies found more activation in the VLFC during maintenance and in the DLFC during manipulation (Fletcher \& Henson, 2001). Moreover, it has recently been suggested that top-down signals from the prefrontal cortex might enhance domain-specific posterior representations (Curtis \& D'Esposito, 2003).

The partial discrepancy between human and nonhuman studies can be explained in a number of ways. In previous studies, human participants might have retained visuo-spatial material verbally, thus, obscuring domain-specific dorsal/ventral segregation in the FC. A possible way to avoid this confound may be to combine visual WM tasks with articulatory suppression to prevent verbal rehearsal of the material. Moreover, process- and domain-specific specialization might coexist within the FC but the experimental design currently used in functional neuroimaging studies might just not be sensitive enough to distinguish them. In particular, the separation of manipulation processes into visual and spatial components has been difficult to demonstrate due to the lack of a clear and specific paradigm for the manipulation of ventral information.

\section{Implications for Neuropsychology and Neuroimaging}

The color-mixing paradigm as utilized in our study provides a tool for the study of active manipulation of ventral stream information in WM. Another interesting direction of research could be the investigation of neuropsychological and neuroanatomical correlates of this dissociated manipulation system in patients with frontal lobe damage, and using functional magnetic resonance imaging or other neuroimaging techniques. We expect that, in particular, the neuroimaging application of the color-mixing paradigm, which to our knowledge is the first purely "ventral" nonverbal manipulation task reported in the literature, will help to clarify the issue of domain- or process-specific specialization in the human prefrontal cortex.

\section{METHODS}

\section{Sample}

Thirty-one volunteers (11 men, mean age $27 \pm$ 5.3 years) participated in Experiment 1, 11 in Experiment 2 ( 6 men, mean age $27.5 \pm 2.38$ years), 30 in Experiment 3 (10 men, mean age $27.7 \pm 6$ years), and 22 in Experiment 4 ( 7 men, mean age $24.8 \pm 9.2$ years). Thirty volunteers participated in Experiment 5 (10 men, mean age $26.4 \pm 6.1$ years) and 30 in Experiment 6 (12 men, mean age $26.2 \pm 3.5$ years). All participants were righthanded, had normal or corrected-to-normal vision, and were tested for normal color vision (Ishihara, 2001).

\section{Experiment 1}

A schematic illustration of the temporal sequence of a trial is given in Figure 1. First, participants were shown two semicircles (samples, $3.1^{\circ} \times 1.34^{\circ}$, separated by $3.98^{\circ}$ center to center) that differed both in spatial orientation and color (500 $\mathrm{msec})$ and then, after a 500-msec delay, an instruction was shown (one of three possible): "mixing color," "mixing rotation angle," or "mixing color and rotation angle" (1500 msec). Then, a white fixation cross $\left(0.022^{\circ} \times 0.022^{\circ}\right)$ was shown for $2000 \mathrm{msec}$ on the screen, followed by a test stimulus in the center of the screen. The test stimulus was a semicircle of a specific color and spatial orientation (presented for $3000 \mathrm{msec}, 3.1^{\circ} \times 1.34^{\circ}$ ). In the colormixing condition, participants had to decide if the color of the test was the same as a mix of the colors of the two samples. In the spatial manipulation condition, partic- 
ipants had to decide if the spatial rotation angle of the test was the same as the average rotation angle of the two samples. In the dual-task condition ("mixing color and rotation angle"), participants had to perform both tasks simultaneously. They were allowed $3 \mathrm{sec}$ to press either the yes or no button. Once the participants had pressed a button, they were given feedback. The fixation cross turned green (1000 msec) if the decision was correct, red if the decision was incorrect, and gray when there was no decision within a time window of 3 sec. The fixation cross (white) was presented in the gap between the trials (2000 $\mathrm{msec})$.

\section{Experiment 2}

Experiment 2 was identical to Experiment 1, with the only exception that there was no gap between the presentation of the samples and the test (Figure 1). The test was presented simultaneously with the samples in the center of the screen below the samples (distance to samples: $4.2^{\circ}$, center to center). Samples and test were presented for $3000 \mathrm{msec}$ and feedback was given immediately afterward (1000 $\mathrm{msec})$. The instruction was shown for 1500 msec before the stimuli. In between the trials, a fixation cross was presented for $2000 \mathrm{msec}$.

\section{Experiment 3}

At the beginning of each trial, the instruction was shown for $1500 \mathrm{msec}$. The task was identical to Experiment 1. Following the instruction, the first sample was shown for $600 \mathrm{msec}$ on the right half of the screen. Participants had to memorize the color or the spatial orientation of the sample or both. After a delay of $400 \mathrm{msec}$, the second sample was shown on the left side of the screen (3500 msec). Simultaneously with the second sample, the test was presented in the center of the screen below the second sample (presentation $3500 \mathrm{msec}$, separated from samples by an angle of $4.2^{\circ}$, center to center) (Figure 1). Participants had 3500 msec to make their decision by pressing the yes or no button. Feedback was given immediately afterward as in Experiments 1 and 2 (1000 msec). In between trials, a fixation cross was shown for $2000 \mathrm{msec}$.

\section{Experiment 4}

Experiment 4 was constructed like Experiment 2, but as a spatial-spatial dual task (Figure 1). First, participants were presented with the instruction (one of three possible, $1500 \mathrm{msec}$ ): "mixing rotation angle," "half distance," and "mixing rotation angle and half distance." Then the test $\left(1.3^{\circ} \times .64^{\circ}\right)$ was presented simultaneously with the samples $\left(1.3^{\circ} \times .64^{\circ}\right)$ in the center of the screen below the samples. Samples and test were presented for 3000 msec and feedback was given immediately afterward (1000 msec). In the "mixing rotation angle" condi- tion, participants had to perform the same rotation task like in the experiments before. In the condition "half distance," participants had to judge if the distance between the test stimulus and the right sample was half of the distance between the two samples (center to center). In the dual-task condition ("mixing rotation angle and half distance"), participants had to do both tasks concurrently. Between the trials, a fixation cross was presented for $2000 \mathrm{msec}$.

\section{Experiment 5}

Experiment 5 examined performance in two manipulation tasks ("mixing color" and "mixing rotation angle," similar to the single conditions of Experiment 1) under several dual-tasks conditions: control (no interference task, NI), interference by articulatory suppression (AS), and interference by random generation (RG). Each condition was presented in two blocks, in which participants had to mix the color or the rotation angle. The order of the blocks was randomized. For the articulatory suppression, participants had to repeat continuously the sound "la" while performing the main tasks. For the random generation task, participants had to generate orally a continuous sequence of letters, but avoid familiar strings. A rate of one letter every $2 \mathrm{sec}$ was required (prompted by beep-tones from the PC every $2 \mathrm{sec}$ ). Each block was preceded by the presentation of the instruction.

\section{Experiment 6}

Experiment 6 differed from Experiment 5 in that participants had to perform two maintenance tasks. They were shown sample stimuli similar to those of Experiment 5 and instructed to memorize either the color or orientation of the two semicircles. In contrast to Experiment 5, the fixation cross was not followed by just one but two test stimuli at the same locations as the samples (Figure 4). Participants had $3000 \mathrm{msec}$ to decide if the colors of the two tests matches the colors of the two samples (maintenance of color) or if the rotation angles of the two tests were identical to the rotation angle of one of the two samples (yes or no button press + feedback $1000 \mathrm{msec}$ ).

\section{Materials and Stimuli}

The stimuli were presented on a 19-in. multiscan 20 SE monitor (Sony Electronics, Tokyo, Japan) with a dark background $\left(0.26 \mathrm{~cd} / \mathrm{m}^{2}\right)$. The monitor was calibrated with a photometer (Minolta CS-100, Konica Minolta Holdings, Inc., Tokyo, Japan). The experimental room was dark except for the light emitted by the monitor.

In the spatial rotation task, the sample stimuli were rotated clockwise around their center. One sample differed from the other by a rotation of $30^{\circ}(50 \%)$ or $40^{\circ}$ 
(50\%). In the matching conditions of Experiments 1-5 (manipulation), the rotation angle of the test $\left(X_{1}\right)$ was the average angle of the two sample rotation angles $\left(X_{2}\right.$ and $\left.X_{3}\right): X_{1}=\left(X_{2}+X_{3}\right) / 2$. The nonmatching test angles differed from the average angle by $\pm 10^{\circ}(8.3 \%)$, $15^{\circ}(33.3 \%), 20^{\circ}(16.6 \%), 35^{\circ}(16.6 \%)$, or $40^{\circ}(16.6 \%)$. In the matching condition of Experiment 6 (maintenance), the rotation angles of the two test stimuli were equal to the rotation angle of one of the two samples. In the nonmatching condition, the test angles differed from both samples.

In the spatial distance task, the distance between the samples was varied in 12 steps between $2.74^{\circ}$ and $5.9^{\circ}$ of visual angle. The test stimulus was presented below the right sample. In the matching condition, the distance from the test to the right sample was half of the distance between the two samples (Figure 1). In the nonmatching condition, the distance between test stimulus and right sample stimulus differed from the bisection of the sample-to-sample distance by $0.64^{\circ}$ or $0.55^{\circ}$.

The colors of the pairs of samples and the test were matched in brightness and saturation, according to the CIE 1931 standard observer for photopic luminous, $V_{\ddot{e}}$. Only the hues or dominant wavelengths of the stimuli were allowed to be deviant. For Experiments 1-4, the perceived combination (test) of the two colors (samples) is located in the middle of the color space between the two colors (CIE 1976, L* $\mathrm{u}^{*} \mathrm{v}^{*}$ ). The CIE $1976 \mathrm{~L}^{*} \mathrm{u}^{*}$ $\mathrm{V}^{*}$ color space was chosen because equally large differences in perceptual color space are assumed to correspond to almost equal distances in the CIE $1976 \mathrm{~L}^{*} \mathrm{u}^{*} \mathrm{v}^{*}$ color space, which is not the case in the CIE 1931 version (Wyszecki \& Stiles, 2000). The hue differences were defined by:

$$
\Delta H^{*}{ }_{u v}=\left[\left(\Delta E_{u v}\right)^{2}-\left(\Delta L_{w v}\right)^{2}-\left(\Delta C_{u v}\right)^{2}\right]^{1 / 2}
$$

If $H$ is the hue difference for two colors in the $\mathrm{u}^{*} \mathrm{v}^{*}$ coordinates, $E$ is the total color difference, $L$ is the difference in lightness, and $C$ is the difference in chroma (Wyszecki \& Stiles, 2000). Fourteen sample color pairs and 14 mixed colors (test) were constructed. Fifty-six colors were designed as tests for the nonmatching conditions. For the 14 mixed colors, a validation was carried out to ensure that the calculated intermediate colors were in fact perceived as intermediate colors. Thirty-two participants rated on a scale from 0 to 4 how well a presented color fitted the ideal mixed color $(0=$ "clearly not the mixed color"; 4 = "definitely the mixed color").

The participants rated the "calculated" colors highest (mean $=3.2, S D=0.45$ ). Other colors that were located in the color space between the two sample colors, although not exactly in the calculated middle, were rated significantly lower than the calculated mixed colors (mean $=0.6, S D=0.61, t=20.9, p<.01$ ). In Experiment 6, the same 14 sample color pairs as in the other experiments were used. In the matching condition, the test colors were identical to the samples; in the nonmatching condition, one of the two test colors was either the mixed color of the two samples $(50 \%)$ or it was located in the color space between the mixed color and one of the sample colors (50\%).

\section{Data Collection}

For each participant, 108 trials were presented in Experiments 1, 2, and 4-6 and 126 in Experiment 3, yielding 36 for each condition (42 in Experiment 3). In every condition, 24 (66.6\%) nonmatching and 12 (33.3\%) matching tests were presented (28 nonmatching and 14 matching for Experiment 3). Trials were presented in random order. The monitor was turned on 20 min before the start of each session to achieve constant monitor temperatures throughout the experiment.

\section{Acknowledgments}

We thank Michael Wibral and Prof. Wolf Singer for helpful discussions that led to the design of Experiment 3, Christoph Bledowski and Alexander T. Sack for suggestions on statistical procedures, Vincent van de Ven, Dr. Corinna Haenschel, and Prof. Ruxandra Sireteanu for comments on the manuscript, and Prof. Konrad Maurer for his constant support. Prof. Ute Leonards provided very helpful advice on the calibration of color stimuli. The Institute of Biophysics of Technische Universität Darmstadt kindly allowed us the use of their photometer. The authors gratefully acknowledge support by the Mordhorst Foundation and Alzheimer Forschung Initiative.

Reprint requests should be sent to David E. J. Linden, School of Psychology, University of Wales Bangor, Brigantia Building, Penrallt Road, Bangor LL57 2AS, UK, or via e-mail: d.linden@ bangor.ac.uk.

\section{REFERENCES}

Baddeley, A. D. (1966). The capacity for generating information by randomization. Quarterly Journal of Experimental Psychology, 18, 119-129.

Baddeley, A. D. (1992). Working memory. Science, 255, 556-559.

Baddeley, A. D. (1996). Exploring the central executive. Quarterly Journal of Experimental Psychology, 49A, 5-28.

Baddeley, A. D., \& Hitch, G. (1974). Working memory. In G. A. Bower (Ed.), Recent advances in learning and motivation (vol. 8, pp. 47-90). New York: Academic Press.

Bruyer, R., \& Scailquin, J. C. (1998). The visuospatial sketchpad for mental images: Testing the multicomponent model of working memory. Acta Psychologica, 98, 17-36.

Carlesimo, G. A., Perri, R., Turriziani, P., Tomaiuolo, F., \& Caltagirone, C. (2001). Remembering what but not where: Independence of spatial and visual working memory in the human brain. Cortex, 37, 519-534.

CIE Proceedings 1931. (1932). Cambridge: Cambridge University Press.

Cornoldi, C., Rigoni, F., Venneri, A., \& Vecchi, T. (2000). Passive and active processes in visuo-spatial memory: 
Double dissociation in developmental learning disabilities. Brain and Cognition, 43, 117-120.

Courtney, S. M., Petit, L., Haxby, J. V., \& Ungerleider, L. G. (1998). The role of prefrontal cortex in working memory: Examining the contents of consciousness. Philosophical Transactions of the Royal Society of London: B. Biological Sciences, 353, 1819-1828.

Curtis, C. E., \& D'Esposito, M. (2003). Persistent activity in the prefrontal cortex during working memory. Trends in Cognitive Sciences, 7, 415-423.

Della Sala, S., Gray, C., Baddeley, A. D., Allamano, N., \& Wilson, L. (1999). Pattern span: A tool for unwelding visuo-spatial memory. Neuropsychologia, 37, 1189-1199.

D'Esposito, M., Postle, B. R., \& Rypma, B. (2000). Prefrontal cortical contributions to working memory: Evidence from event-related fMRI studies. Experimental Brain Research, 133, 3-11.

Donaldson, W. (1993). Accuracy of $d^{\prime}$ and $A^{\prime}$ as estimates of sensitivity. Bulletin of the Psychonomic Society, 31, 271-274.

Farah, M. J., Hammond, K. M., Levine, D. N., \& Calvanio, R. (1988). Visual and spatial mental imagery: Dissociable systems of representation. Cognitive Psychology, 20, 439-462.

Fletcher, P. C., \& Henson, R. N. A. (2001). Frontal lobes and human memory. Insights from functional neuroimaging. Brain, 124, 849-881.

Formisano, E., Linden, D. E., Di Salle, F., Trojano, L., Esposito, F., Sack, A. T., Grossi, D., Zanella, F. E., \& Goebel, R. (2002). Tracking the mind's image in the brain I: Time-resolved fMRI during visuospatial mental imagery. Neuron, 35, 185-194.

Goebel, R., Khorram-Sefat, D., Muckli, L., Hacker, H., \& Singer, W. (1998). The constructive nature of vision: Direct evidence from functional magnetic resonance imaging studies of apparent motion and motion imagery.

European Journal of Neuroscience, 10, 1563-1573.

Goldman-Rakic, P. S. (1987). Circuitry of primate prefrontal cortex and regulation of behavior by representational memory. In V. B. Mountcastle \& F. Plum (Eds.), Handbook of physiology (section 1, vol. 5, pp. 373-417). Bethesda, MD: American Physiological Society.

Goldman-Rakic, P. S. (1996). The prefrontal landscape: Implications of functional architecture for understanding human mentation and the central executive. Philosophical Transactions of the Royal Society of London, Series B, Biological Sciences, 351, 1445-1453.

Goodale, M. A. (1998). Vision for perception and vision for action in the primate brain. In R. G. Bock, \& J. A. Goode (Eds.), Sensory guidance of movement. Novartis Foundation Symposium Series (vol. 218, pp. 21-34). Chichester: Wiley.

Grier, J. B. (1971). Nonparametric indexes for sensitivity and bias: Computing formulas. Psychological Bulletin, 75, 424-429.

Hecker, R., \& Mapperson, B. (1997). Dissociation of visual and spatial processing in working memory. Neuropsychologia, 35, 599-603.

Ishihara, M. D. (2001). Ishibara's tests for colour deficiency. Tokyo: Kanehara \& Co.

Kessels, R. P., Postma, A., \& de Haan, E. H. (1999). P and M channel-specific interference in the what and where pathway. NeuroReport, 10, 3765-3767.

Levy, B. A. (1977). Reading: Speech and meaning processes. Journal of Verbal Learning and Verbal Behavior, 16, 623-638.

Livingstone, M. S., \& Hubel, D. H. (1987). Psychophysical evidence for separate channels for the perception of form, color, movement, and depth. Journal of Neuroscience, 7 , 3416-3468.

Logie, R. H. (1995). Visuo-spatial working memory. Hove: Erlbaum.

Logie, R. H., \& Marchetti, C. (1991). Visuo-spatial working memory: Visual, spatial or central executive? In R. H. Logie \& M. Denis (Eds.), Mental images in buman cognition (pp. 105-115). Amsterdam: North-Holland Press.

Luzzatti, C., Vecchi, T., Agazzi, D., Cesa-Bianchi, M., \& Vergani, C. (1998). A neurological dissociation between preserved visual and impaired spatial processing in mental imagery. Cortex, 34, 461-469.

Meegan, D. V., Purc-Stephenson, R., Honsberger, M. J., \& Topan, M. (2004). Task analysis complements neuroimaging An example from working memory research. Neuroimage, 21, $1026-1036$.

Morton, N., \& Morris, R. G. (1995). Image transformation dissociated from visuo-spatial working memory. Cognitive Neuropsychology, 12, 767-791.

Munk, M. H., Linden, D. E. J., Muckli, L., Lanfermann, H., Zanella, F. E., Singer, W., \& Goebel, R. (2002). Distributed cortical systems in visual short-term memory revealed by event-related functional magnetic resonance imaging. Cerebral Cortex, 12, 866-876.

Petrides, M. (1994). Frontal lobes and working memory: Evidences from investigations of the effects of cortical excisions in nonhuman primates. In F. Boller, \& J. Grafman (Eds.), Handbook of neuropsychology (pp. 59-82). Amsterdam: Elsevier.

Pickering, S. J. (2001). Cognitive approaches to the fractionation of visuo-spatial working memory. Cortex, 37, 457-473.

Sakata, H., Taira, M., Kusunoki, M., Murata, A., \& Tanaka, Y. (1997). The TINS Lecture. The parietal association cortex in depth perception and visual control of hand action. Trends in Neurosciences, 20, 350-357.

Tresch, M. C., Sinnamon, H. M., \& Seamon, J. G. (1993). Double dissociation of spatial and object visual memory: Evidence from selective interference in intact human subjects. Neuropsychologia, 31, 211-219.

Ungerleider, L. G., Courtney, S. M., \& Haxby, J. V. (1998). A neural system for human visual working memory. Proceedings of the National Academy of Sciences, U.S.A., 95, 883-890.

Ungerleider, L. G., \& Haxby, J. V. (1994). "What" and "where" in the human brain. Current Opinion in Neurobiology, 4 , $157-165$.

Ungerleider, M. G., \& Mishkin, M. (1982). Two cortical visual systems. In D. J. Ingle, M. A. Goodale, \& R. J. W. Mansfield (Eds.), Analysis of visual behavior (pp. 549-586). Cambridge: MIT Press.

Vecchi, T. (1998). Visuo-spatial imagery in congenitally totally blind people. Memory, 6, 91-102.

Vecchi, T., \& Cornoldi, C. (1999). Passive storage and active manipulation in visuo-spatial working memory: Further evidence from the study of age differences. European Journal of Cognitive Psychology, 11, 391-406.

Vecchi, T., \& Girelli, L. (1998). Gender differences in visuo-spatial processing: The importance of distinguishing between passive storage and active manipulation. Acta Psychologica, 99, 1-16.

Vuontela, V., Rama, P., Raninen, A., Aronen, H. J., \& Carlson, S. (1999). Selective interference reveals dissociation between memory for location and colour. NeuroReport, 10, 2235-2240.

Wyszecki, G., \& Stiles, W. S. (2000). Color science concepts and methods, quantitative data and formulae (2nd ed.). New York: Wiley. 